\title{
Síndrome de Mirizzi coloduodenal, propuesta para incluir la variante la, Ib y su manejo en la nueva clasificación de Beltrán
}

\author{
Mirizzi coloduodenal syndrome, proposal for including variant la, Ib and its \\ management in the new Beltrán classification
}

Francisco Amador Rivera López,* Gerardo Antonio Guerrero Márquez,**

Francisco Miñana Echávarri,** Andrés Herman Blancarte Linden**

\begin{abstract}
Palabras clave: Síndrome de Mirizzi, fístula biliar, fístula bilioentérica.

Key words: Mirizzi syndrome, biliary fistula, biliary-enteric fistula.
\end{abstract}

\section{RESUMEN}

Introducción: El síndrome de Mirizzi (SM) describe la obstrucción mecánica del conducto hepático común ocasionada por una compresión extrínseca (con o sin una fístula asociada). Ocurre aproximadamente entre 0.2 y $1.5 \%$ de los pacientes con litiasis vesicular. La inflamación continua de la vesícula biliar puede evolucionar a una compresión y fusión de vísceras como colon y duodeno. Los pacientes tienen una larga historia de litiasis vesicular y los síntomas que presentan son inespecíficos. Las metas del tratamiento son descomprimir la vía biliar mediante cualquier método y prevenir la recurrencia. El tratamiento quirúrgico se basa en la clasificación de Beltrán y en los principios de Culture of Safety in Cholecistectomy (COSIC). Reporte de caso: Se presenta el caso de una paciente programada para colecistectomía laparoscópica por colelitiasis, pero se decidió conversión a cirugía abierta debido a la importante fusión biliar inflamatoria hacia colon y duodeno y a la mala identificación de estructuras sin poder lograr la visión crítica de seguridad. Fue manejada mediante sonda de colecistostomía para posteriormente planear el tratamiento quirúrgico definitivo. Conclusión: El caso presentado con compresión de la vesícula biliar y fusión biliar inflamatoria a colon, duodeno y vías biliares es una variante del SM que no está descrita por la baja frecuencia de su presentación, por lo que proponemos que se incluyan el tipo Ia y el Ib del SM en la clasificación de Beltrán para disminuir el riesgo de lesión vasculobiliar e intestinal mediante los principios de COSIC, con recomendación de colecistostomía como tratamiento quirúrgico para el SM tipo Ib.

\section{ABSTRACT}

Introduction: The Mirizzi syndrome (SM) is used to describe the mechanical obstruction of the common hepatic duct due to extrinsic compression (with or without associated fistula). It occurs in approximately 0.2 to $1.5 \%$ of patients with cholelithiasis. The continued inflammation of the gallbladder can evolve into a compression and fusion to adjacent viscera like colon and duodenum. Patients have a long history of gallstone disease and symptoms that are nonspecific. The goals of treatment are to decompress the bile duct and prevent recurrence. Surgical treatment is based on the classification of Beltran and Culture of Safety in Cholecistectomy (COSIC) principles. Case report: We present the case of a patient scheduled for laparoscopic cholecystectomy for cholelithiasis, where conversion to open by the major bile inflammatory fusion to colon and duodenun and misidentification of structures, managed with a cholecystostomy tube, in order to, later planning definitive surgical treatment. Conclusion: The case presented with compression of the gallbladder and inflammatory biliary fusion colon duodenum and bile ducts is a variant of SM that is not described by the rarity of presentation we propose to add to the classification of Beltran SM type Ia and Ib to reduce the risk of vasculobiliar and intestinal by principles of COSIC we recommending colcistostomy as surgical treatment for type Ib SM.

\footnotetext{
* Titular del Curso de Cirugía General.

** Médico Residente de Cirugía General.
}

Instituto Mexicano del Seguro Social. Hospital General de Zona con Medicina Familiar número 16, Torreón Coahuila. Aceptado: 31/10/2017

\section{INTRODUCCIÓN}

\section{Síndrome de Mirizzi (SM)}

A unque Hans Kehr ${ }^{1}$ en 1905 fue el primero en reportar una obstrucción parcial de la vía biliar debida a un lito impactado en la vesícula biliar, fue el artículo Syndrome du canal hepatique ${ }^{2}$ de Pablo Luis Mirizzi en 1948 el que llamó la atención sobre esta patología. 


\section{Fístula biliar}

Las fístulas biliares son una comunicación anormal del sistema biliar a una localización interna o externa. Este padecimiento comparte la fisiopatología con la obstrucción biliar, infección, necrosis y fistulización. Las fístulas internas pueden formarse en el tracto gastrointestinal, cavidad torácica, estructuras genitourinarias o sistemas vasculares. Las fístulas externas son comunicaciones del sistema biliar a la piel, ya sea espontáneamente o secundarias a la intervención quirúrgica durante una colecistectomía en la que hay salida de bilis por la herida quirúrgica o en el sitio de drenaje. La obstrucción del tubo digestivo puede resultar del paso de grandes litos a través de fístulas bilioentéricas con lito impactado en el intestino delgado o colon (íleo biliar) o en la región pilórica (síndrome de Bouveret). También puede ocurrir una fistulización entre la vesícula biliar y la vía biliar extrahepática (SM). Aunque son raros, estos padecimientos pueden causar morbilidad y mortalidad significativas que requieren atención. $^{3}$

\section{Fisiopatología}

El SM se utiliza para describir la obstrucción mecánica del conducto hepático común debido a una compresión extrínseca (con o sin una fístula asociada). La obstrucción es ocasionada por lo regular por un lito de gran tamaño impactado en el cuello o infundíbulo de la vesícula biliar adyacente a la vía biliar extrahepática. Algunas veces la obstrucción extrínseca es causada por litos pequeños impactados en el conducto cístico que tiene un trayecto paralelo al conducto hepático común o incluso por la misma vesícula biliar distendida a tensión. Mirizzi postuló en su artículo $^{2}$ de manera incorrecta que un espasmo del conducto hepático común era la causa de la obstrucción de la vía biliar. El SM ocurre aproximadamente entre 0.2 y $1.5 \%$ de los pacientes con litiasis vesicular. Beltrán teorizó que la compresión externa de la vía biliar y el posterior desarrollo de una fístula colecistobiliar y colecistoentérica son diferentes estadios del mismo proceso patológico. ${ }^{4}$

\section{Clasificación}

Los esquemas de clasificación se basan en la presencia o ausencia de erosiones fistulosas entre la vesícula biliar y la vía biliar común y la extensión de la destrucción de esta última.

La clasificación y caracterización del SM permaneció sin cambios hasta 1982 cuando McSherry y cols. ${ }^{5}$ subclasificaron el SM con base en los resultados de la colangiopancreatografía retrógrada endoscópica (CPRE) y encontraron que una presión constante desde dentro de la vesícula puede crear comunicaciones fistulosas entre ésta y el árbol biliar.

La clasificación original de McSherry describió dos tipos de SM:

- Tipo I: compresión de la vía biliar común por un lito impactado en el conducto cístico o bolsa de Hartmann.

- Tipo II: erosión del cálculo del conducto cístico en el conducto hepático común o el conducto biliar común, la producción de una fístula colecisto-coledociana.

Csendes en $1989^{6}$ expandió la clasificación en cuatro grados:

- Tipo I: compresión externa de la vía biliar común debida a un cálculo impactado en el cuello de la vesícula biliar o en el conducto cístico.

- Tipo II: presencia de una fístula colecistobiliar (colecistohepática o colecisto-coledociana) causada por la erosión de la pared anterior o lateral de la vía biliar común por un lito impactado que afecta menos de un tercio de la circunferencia de la vía biliar común.

- Tipo III: fístula colecistobiliar con erosión de la pared del conducto biliar común que afecta más de dos tercios de la circunferencia.

- Tipo IV: fístula colecistobiliar con destrucción completa de la pared de la vía biliar común.

La historia natural del SM no termina con la formación de la fístula colecistobiliar, la inflamación continua del área del triángulo de Calot no sólo puede formar una fístula compleja que afecta el tracto biliar, sino también vísceras adyacentes. Basados en esta suposición, Csen- 
des y cols. recientemente agregaron un nuevo tipo a su clasificación que incluye la fístula colecistoentérica, complicando a los otros tipos.

En la nueva clasificación propuesta en 2008 se agrega el Mirizzi tipo $\vee$ y dos subtipos: ${ }^{7}$

- Tipo V: cualquier tipo de fístula colecistobiliar más una fístula colecistoentérica.

- Tipo Va: sin íleo biliar.

- Tipo Vb: con íleo biliar.

\section{Presentación clínica}

Los pacientes con SM por lo general son mujeres entre la quinta y séptima década de

Cuadro I. Nueva clasificación y manejo

quirúrgico sugerido para el síndrome de Mirizzi.

\begin{tabular}{|c|c|c|}
\hline Tipo de Mirizzi & Descripción & Tratamiento \\
\hline I & $\begin{array}{l}\text { Compresión externa del } \\
\text { conducto biliar }\end{array}$ & $\begin{array}{l}\text { Colecistectomía abierta } \\
\text { Colecistectomía subtotal } \\
\text { abierta } \\
\text { Colecistectomía } \\
\text { laparoscópica } \\
\text { Colecistectomía subtotal } \\
\text { laparoscópica }\end{array}$ \\
\hline IIa & $\begin{array}{l}\text { Fístula colecistobiliar de } \\
<50 \% \text { de diámetro del } \\
\text { conducto biliar }\end{array}$ & $\begin{array}{l}\text { Colecistectomía abierta } \\
\text { Colecistectomía abierta } \\
\text { subtotal }\end{array}$ \\
\hline IIb & $\begin{array}{l}\text { Fístula colecistobiliar de } \\
\text { > 50\% de diámetro del } \\
\text { conducto biliar }\end{array}$ & $\begin{array}{l}\text { Colecistectomía subtotal } \\
\text { abierta } \\
\text { Derivación biliodigestiva: } \\
\text { Colédoco-duodenostomía } \\
\text { Colédoco-yeyunostomía } \\
\text { en Y de Roux }\end{array}$ \\
\hline IIIa & $\begin{array}{l}\text { Fístula colecistobiliar y } \\
\text { fístula colecistoentérica } \\
\text { sin íleo biliar }\end{array}$ & $\begin{array}{l}\text { Cierre simple de la } \\
\text { fístula colecistoentérica } \\
\text { y tratamiento de acuerdo } \\
\text { a la presencia de Mirizzi } \\
\text { I, IIa o IIb para la fístula } \\
\text { colecistobiliar }\end{array}$ \\
\hline IIIb & $\begin{array}{l}\text { Fístula colecistobiliar y } \\
\text { fístula colecistoentérica } \\
\text { con íleo biliar }\end{array}$ & $\begin{array}{l}\text { Tratamiento del íleo biliar } \\
\text { y tratamiento diferido de } \\
\text { acuerdo con la presencia } \\
\text { de Mirizzi I, IIa o IIb para } \\
\text { la fístula colecistobiliar }\end{array}$ \\
\hline
\end{tabular}

la vida, tienen una larga historia de litiasis vesicular y los síntomas que presentan son de ictericia obstructiva, además los pacientes con SM pueden mostrar un cuadro de colecistitis aguda, colangitis o pancreatitis.

\section{Diagnóstico}

El diagnóstico preoperatorio de SM puede ser difícil, pero una planeación quirúrgica cuidadosa y un reconocimiento transoperatorio pueden evitar la alta incidencia de lesión de vía biliar. ${ }^{8}$ La tomografía y el ultrasonido no son específicos, pero pueden ser sugestivos cuando se detecta dilatación de la vía biliar intrahepática o hepática común proximal. La certeza de la CPRE es de 55 a 90\%. Debido a la dificultad del diagnóstico preoperatorio más de la mitad de los casos de SM no se diagnostican hasta la cirugía. ${ }^{9}$ Los hallazgos característicos durante la cirugía son fibrosis y obliteración de las estructuras del triángulo de Calot a causa de litos impactados en el infundíbulo o conducto cístico. La vesícula biliar puede estar contraída o dilatada con engrosamiento en la pared. La colangiografía transoperatoria y el ultrasonido son herramientas útiles para delinear la anatomía anormal en medio de la fibrosis y la inflamación.

\section{Tratamiento}

Las metas de tratamiento para los pacientes con SM son descomprimir la vía biliar y prevenir la recurrencia. Se ha recurrido a la colocación de stents en la vía biliar temporalmente durante la CPRE para ganar tiempo y permitir un tratamiento médico óptimo y la planeación quirúrgica adecuada.

El tratamiento quirúrgico está basado en el tipo de Mirizzi, el tipo I puede ser manejado con colecistectomía o colecistectomía subtotal únicamente, mientras que los tipos II, III y IV requieren una reconstrucción biliar. El tratamiento quirúrgico se basa en la nueva clasificación y manejo sugerido por Beltrán para el SM (Cuadro I). ${ }^{10}$ Aunque se ha descrito el manejo laparoscópico para el SM, ${ }^{11}$ los cirujanos deben estar alertas y tener precaución por el alto riesgo de lesión de vía biliar. Cuando es diagnosticado de manera preoperatoria, debe 
considerarse seriamente referir al paciente a un centro con experiencia en cirugía de vía biliar. Debido a la posibilidad de detectar cáncer de vesícula hasta en $25 \%$ de los casos con SM, debe realizarse una sección transoperatoria de cortes por congelamiento de la pared de la vesícula biliar y de la capacidad para disponer de tratamiento oncológico de resección. ${ }^{12}$

\section{CASO CLÍNICO}

Paciente femenino de 52 años de edad, la cual cuenta con los siguientes antecedentes de importancia: niega crónico-degenerativos, quirúrgicos positivos para histerectomía abdominal secundaria a miomatosis hace cuatro años y oclusión tubárica bilateral de intervalo abierta hace 25 años sin complicaciones, niega transfusionales y alérgicos.

Inicia padecimiento siete meses previos a la cirugía con cuadro de dolor abdominal de tipo cólico en hipocondrio derecho irradiado hacia ángulo escapuloapexiano ipsilateral sin síntomas acompañantes, refiere fue su primer cuadro sin presentar recurrencias, niega ictericia, acolia, coluria o fiebre. Cuenta con ultrasonido (04/09/14) que reportó hígado sin alteraciones ecográficas, porta $7 \mathrm{~mm}$, colédoco $3 \mathrm{~mm}$, vesícula biliar distendida $8 \times 3 \times 3$ $\mathrm{cm}$, pared de $2 \mathrm{~mm}$, se observa en su interior imagen ecogénica que mide $27 \mathrm{~mm}$ así como múltiples litos que van de 22 a $24 \mathrm{~mm}$ con sombra acústica posterior en cuerpo y fondo, páncreas sin alteraciones ecográficas y bazo sin alteraciones ecográficas.

Programada para realizar colecistectomía laparoscópica (16/05/15).

Hallazgos transoperatorios: adherencias de epiplón a fondo de vesícula biliar, contracción de vesícula biliar y lecho hepático, el epiplón cubre la fusión biliar de colon, duodeno y vías biliares, se aprecia material purulento entre el epiplón y las estructuras anatómicas antes mencionadas (Figura 1).

Técnica quirúrgica: abordaje laparoscópico con cuatro puertos, después de retirar el epiplón el fondo de la vesícula biliar se encuentra libre, se continúa con disección y separación de la vesícula biliar del colon y el duodeno, pero se despulen con riesgo de perforación, por lo que se decide conversión a cirugía abierta con

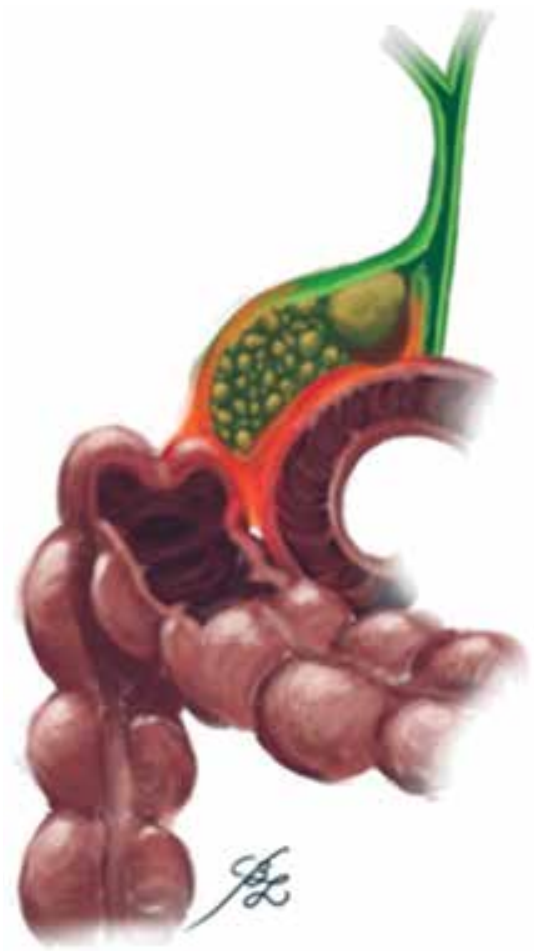

Figura 1. Hallazgos transoperatorios.

abordaje de Masson por planos de la manera habitual, intentando con disección roma y cortante la separación de las estructuras para realizar la vista crítica de seguridad sin conseguirlo, se observa alto riesgo de perforación de colon, duodeno y lesión de la vía biliar junto con sus estructuras vasculares, motivo por el que se decidió abrir la vesícula biliar a través del fondo (colecistostomía), colocando primero una jareta sobre del fondo de la vesícula con vycril 2 ceros, posteriormente abrimos con bisturí, se extraen litos de medianos elementos y uno de $7 \times 4 \mathrm{~cm}$, se introduce el dedo índice hasta la bolsa de Hartmann, se instala sonda Foley $14 \mathrm{Fr}$ a través de la cual se realiza colangiografía transoperatoria (Figura 2), en la que se aprecia paso de medio de contraste a duodeno con integridad de la vía biliar, se deja sonda Foley, se cierra la jareta con vycril 2-0, se saca la sonda por contrabertura junto con drenaje tipo Penrose dirigido al hiato de Winslow, se fijan a la piel con prolene 2-0, se cierra por planos, aponeurosis con vycril del uno y surget continuo, tejido celular subcutáneo con vycril 2-0 con puntos separados y piel 


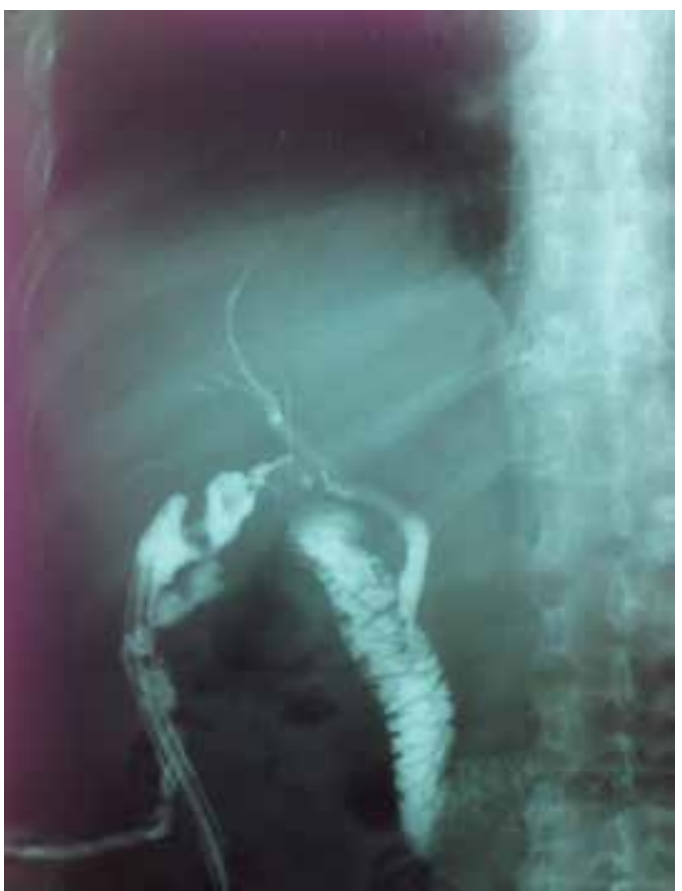

Figura 2. Colangiografía transoperatoria con sonda Foley. Se aprecia defecto de llenado en el cuerpo de vesícula por el globo y otro defecto de llenado en bolsa de Hartmann, compresión de hepático común, compresión a duodeno y colon.

con puntos de Sarnoff con prolene 2-0, dando por terminada la cirugía.

Evolución postoperatoria: alta al cuarto día sin complicaciones.

\section{DISCUSIÓN}

En el SM tipo I existe una compresión de la vía biliar común por un lito impactado en el conducto cístico o bolsa de Hartmann, al continuar el proceso inflamatorio se producen adherencias (SM tipo la) y posteriormente fusión inflamatoria (SM tipo lb) del cuerpo de la vesícula, conducto cístico y bolsa de Hartmann a colon duodeno y vías biliares como en el caso que nos ocupa, estas etapas la y lb se presentan antes de la fístula colecistobiliar (SM tipo Ila y IIb) o colecistoentérica (SM tipo IIla y IIIb) de la clasificación propuesta (Figura 3).

En el SM tipo la las adherencias pueden separarse identificando la vista crítica de Strasberg sin lesionar los órganos involucrados. En la siguiente etapa Ib las adherencias se convierten
A

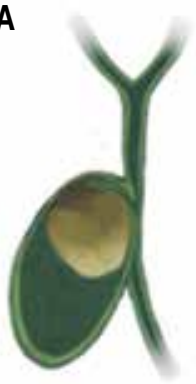

C

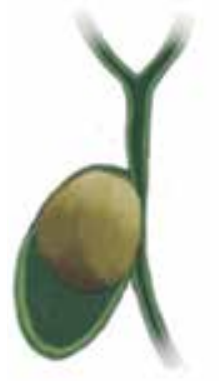

B

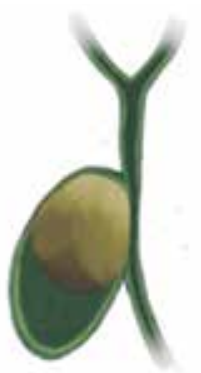

D

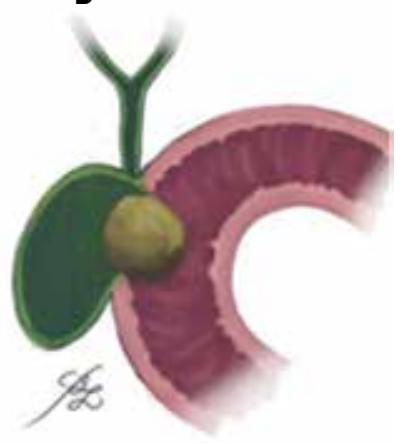

Figura 3. Clasificación propuesta para el manejo quirúrgico del SM: A. SM Ia compresión externa del conducto biliar (fusión biliar inflamatoria y adherencia a órganos y estructuras adyacentes), los cuales pueden disecarse adecuadamente, SM Ib compresión externa del conducto biliar (fusión biliar inflamatoria y adherencia a órganos y estructuras adyacentes), los cuales no pueden disecarse adecuadamente, B. SM IIa fistula colecistobiliar de $<50 \%$ de diámetro del conducto biliar, C. SM IIb fistula colecistobiliar de $>50 \%$ de diámetro del conducto biliar, D. SM IIIa fistula colecistobiliar y fistula colecistoentérica sin íleo biliar, SM IIIb fístula colecistobiliar y fístula colecistoentérica con íleo biliar.

en una fusión inflamatoria del cuerpo de la vesícula, conducto cístico y bolsa de Hartmann a colon, duodeno y vías biliares que no es posible identificar porque al disecar para separar se produce primero despulimiento y si se continúa disecando hay lesión (ruptura) de las estructuras anatómicas involucradas, sin identificar la vista crítica con alto riesgo de lesión vasculobiliar extrema ${ }^{13} \mathrm{y} / \mathrm{o}$ intestinal.

Recalcamos lo importante de no insistir en disecar estructuras fusionadas. La cultura de la seguridad en colecistectomía $(\mathrm{COSIC})^{14}$ no es un método de disección, es de identificación y al no identificar por qué no es posible separar 
Cuadro II. Clasificación y manejo

propuesto para el síndrome de Mirizzi.

\begin{tabular}{|c|c|c|}
\hline Tipo de Mirizzi & Descripción & Tratamiento \\
\hline Ia & $\begin{array}{l}\text { Compresión externa del } \\
\text { conducto biliar (fusión biliar } \\
\text { inflamatoria y adherencia a } \\
\text { órganos y estructuras adya- } \\
\text { centes), los cuales pueden } \\
\text { disecarse adecuadamente }\end{array}$ & $\begin{array}{l}\text { Colecistectomía abierta } \\
\text { Colecistectomía subtotal } \\
\text { abierta } \\
\text { Colecistectomía } \\
\text { laparoscópica } \\
\text { Colecistectomía subtotal } \\
\text { laparoscópica }\end{array}$ \\
\hline $\mathrm{Ib}$ & $\begin{array}{l}\text { Compresión externa del } \\
\text { conducto biliar (fusión bi- } \\
\text { liar inflamatoria y adheren- } \\
\text { cia a órganos y estructuras } \\
\text { adyacentes), los cuales no } \\
\text { pueden disecarse adecuada- } \\
\text { mente }\end{array}$ & $\begin{array}{l}\text { Colecistectomía abierta } \\
\text { Colecistectomía abierta } \\
\text { subtotal } \\
\text { Colecistostomía }\end{array}$ \\
\hline IIa & $\begin{array}{l}\text { Fístula colecistobiliar de < } \\
50 \% \text { de diámetro del con- } \\
\text { ducto biliar }\end{array}$ & $\begin{array}{l}\text { Colecistectomía abierta } \\
\text { Colecistectomía abierta } \\
\text { subtotal } \\
\text { Colecistostomía }\end{array}$ \\
\hline IIb & $\begin{array}{l}\text { Fístula colecistobiliar de > } \\
50 \% \text { de diámetro del con- } \\
\text { ducto biliar }\end{array}$ & $\begin{array}{l}\text { Colecistectomía subtotal } \\
\text { abierta } \\
\text { Colecistostomía } \\
\text { Derivación biliodigestiva: } \\
\text { Coledoco-duodenostomía } \\
\text { Coledoco-yeyunostomía } \\
\text { en Y de Roux }\end{array}$ \\
\hline IIIa & $\begin{array}{l}\text { Fístula colecistobiliar y } \\
\text { fístula colecistoentérica sin } \\
\text { íleo biliar }\end{array}$ & $\begin{array}{l}\text { Cierre simple de la fístula } \\
\text { colecistoentérica y tra- } \\
\text { tamiento de acuerdo con } \\
\text { la presencia de Mirizzi } \\
\text { I, IIa o IIb para la fístula } \\
\text { colecistobiliar }\end{array}$ \\
\hline lllb & $\begin{array}{l}\text { Fístula colecistobiliar y } \\
\text { fístula colecistoentérica con } \\
\text { íleo biliar }\end{array}$ & $\begin{array}{l}\text { Tratamiento del íleo biliar } \\
\text { y tratamiento diferido de } \\
\text { acuerdo con la presencia } \\
\text { de Mirizzi I, IIa o IIb para } \\
\text { la fístula colecistobiliar }\end{array}$ \\
\hline
\end{tabular}

las estructuras anatómicas sin lesionarlas, lo más adecuado es abrir el fondo de la vesícula biliar para extraer los litos y realizar palpación digital y colangiografía transoperatoria, delimitando la anatomía para realizar una colecistectomía subtotal, pero de no ser posible, lo más prudente es realizar una colecistostomía y más tarde, en el postoperatorio, una colangiorresonancia o colangiografía para delimitar la anatomía de la vía biliar y su relación con el tubo digestivo y vasos importantes. Una vez desinflamada el área fusionada se programa la reintervención quirúrgica para proceder a colecistectomía subtotal o para dejar la vesícula biliar fenestrada, lo que impide nueva formación de litos.

\section{CONCLUSIONES}

El caso presentado anteriormente con compresión de la vesícula biliar y fusión biliar inflamatoria a colon, duodeno y vías biliares es una variante del SM que no está descrita por la baja frecuencia de su presentación en la nueva clasificación de Beltrán. Proponemos que el tipo la y el Ib del SM se incluyan en esta nueva clasificación (Cuadro II), de tal forma que cuando se presente esta variante del SM disminuya el riesgo de lesión vasculobiliar e intestinal mediante los principios de COSIC, con recomendación de colecistostomía como tratamiento quirúrgico para el SM tipo Ib.

\section{REFERENCIAS}

1. Kehr H. Die in neiner klinik geubte technik de gallenstein operationen, mit einen hinweis auf die indikationen und die dauerersolge. Munchen (Germany): JF Lehman; 1905.

2. Mirizzi P. Syndrome du canal hepatique. J Int Chir. 1948; 8: 731-733.

3. Luu MB, Deziel DJ. Unusual complications of gallstones. Surg Clin North Am. 2014; 94: 377-394.

4. Beltran MA, Csendes A. Mirizzi syndrome and gallstone ileus: an unusual presentation of gallstone disease. J Gastrointest Surg. 2005; 9: 686-689.

5. McSherry CK, Ferstenberg H, Virshup M. The Mirizzi syndrome: suggested classification and surgical therapy. Surg Gastroenterol. 1982; 1: 219-225.

6. Csendes A, Díaz JC, Burdiles P, Maluenda F, Nava O. Mirizzi syndrome and cholecystobiliary fistula: a unifying classification. Br J Surg. 1989; 76: 1139-1143.

7. Beltran MA, Csendes A, Cruces KS. The relationship of Mirizzi syndrome and cholecystoenteric fistula: validation of a modified classification. World J Surg. 2008; 32: 2237-2243.

8. Lai EC, Lau WY. Mirizzi syndrome: history, present and future development. ANZ J Surg. 2006; 76: 251-257.

9. Safioleas $M$, Stamatakos $M$, Safioleas P, Smyrnis A, Revenas C, Safioleas C. Mirizzi Syndrome: an unexpected problem of cholelithiasis. Our experience with 27 cases. Int Semin Surg Oncol. 2008; 5: 12.

10. Beltrán MA. Mirizzi syndrome: history, current knowledge and proposal of a simplified classification. World J Gastroenterol. 2012; 18: 4639-4650. 
11. Yeh CN, Jan YY, Chen MF. Laparoscopic treatment for Mirizzi syndrome. Surg Endosc. 2003; 17: 15731578.

12. Redaelli CA, Büchler MW, Schilling MK, Krähenbühl L, Ruchti C, Blumgart LH et al. High coincidence of Mirizzi syndrome and gallbladder carcinoma. Surgery. 1997; 121: 58-63.

13. Strasberg SM, Gouma DJ. 'Extreme' vasculobiliary injuries: association with fundus-down cholecystectomy in severely inflamed gallbladders. HPB (Oxford). 2012; 14: 1-8.
14. Strasberg SM. Biliary injury in laparoscopic surgery: part 2. Changing the culture of cholecystectomy. J Am Coll Surg. 2005; 201: 604-11.

\section{Correspondencia:}

Dr. Francisco Amador Rivera López

Bulevar Revolución s/n, esquina con Jazmines,

Col. Torreón Jardín, 27200,

Torreón, Coahuila.

Teléfono: 871-137-4883

E-mail: farl111@yahoo.com.mx 